\title{
Shielding characteristics of water
}

\author{
A.A. Levchenko ${ }^{1}$, A.M. Likhter ${ }^{2}$, D.A. Khramov ${ }^{1,2}$, V.A. Gryaznova ${ }^{2}$, and V.B. Shikin ${ }^{1}$ \\ ${ }^{1}$ Institute of Solid State Physics RAS, Chernogolovka 142432, Moscow District, Russia \\ ${ }^{2}$ Astrakhan State University, Astrakhan 414056, Russia \\ E-mail: levch@issp.ac.ru
}

Received January 28, 2015, published online April 23, 2015

\begin{abstract}
In this article we demonstrate that a relatively small density of intrinsic ions in pure water has a significant impact on the development of the instability of the liquid-vapor boundary in an external electric field perpendicular to the interface. Dielectric breakdown scenario (Shliomis model) is shunted by alternative metal scenario (Frenkel model). Experimentally we observed the formation of a positively charged layer beneath the surface in weak perpendicular electrical fields. In strong electrical fields surface of water loses its stability and charges pass through the interface. Surface discharge process is periodic with a characteristic time of the order of tens seconds.
\end{abstract}

PACS: 52.20.-j Elementary processes in plasmas.

Keywords: water, electric properties, stability of liquid surface, dissociation, charges.

\section{Introduction}

The problem of stability of a boundary for two liquid dielectric media was considered in [1,2]. Let us consider this problem when the degree of water dissociation $\alpha_{\mathrm{aq}}=0$. For a vacuum-liquid boundary with dielectric constant $\varepsilon$ the stability threshold is defined by the expression $E_{0}=E_{\varepsilon}[1]$ where

$$
E_{\varepsilon}^{2}=8 \pi \frac{\varepsilon(\varepsilon+1)}{(\varepsilon-1)^{2}} \sqrt{\rho g \gamma}, \quad k_{0}=\sqrt{\rho g / \gamma} .
$$

Here $\rho$ is the liquid density, $k_{0}$ is the minimal wave number in stability problem, $\gamma$ the surface tension of the vapor-liquid interface, $g$ is the gravitational acceleration. The vacuum region is in the upper half-plane, the normal part of field $E_{0}$ in vacuum is related to the field inside the liquid on the oscillating surface by condition $E_{0}=\varepsilon E_{\text {insid }}$.

Formula (1) is valid for any liquid with parameters $\rho, \varepsilon, \gamma$. In particular, at $\varepsilon \rightarrow 1$ (cryogenic media), critical field $E_{\varepsilon}(\varepsilon \rightarrow 1) \rightarrow \infty$ (in the case of $\varepsilon \rightarrow 1$ the vacuumliquid boundary in field $E_{0}$ ceases to be a source of ponderomotive forces of dielectric origin). In the inverse limit $\varepsilon \rightarrow \infty$, i.e., the dielectric critical field tends to the metallic limit, $E_{\varepsilon} \rightarrow E_{\text {met }}$,

$$
E_{\text {met }}^{2}=8 \pi \sqrt{\rho g \gamma} .
$$

In this scenario field $E_{\text {insid }}$ inside the liquid becomes zero (the influence of boundary condition $E_{0}=\varepsilon E_{\text {insid }}$ ), which is typical for good conductors. Expression (2) for stability threshold of good conducting liquid surface was obtained eighth decades earlier by Frenkel [3,4].

The presence of low but finite dissociation $\alpha \neq 0$ has a significant effect on electric properties of water. In the regime with a constant but weakly time-dependent field $E_{0}(t)$ (smoothness is required for accurate attainment of the instability threshold) the liquid charges are spatially separated to form an accumulation layer of thickness $\lambda$ near the walls of the cup with water and gradually charge free surface. As is known, charge accumulation on the vacuum-electrolyte boundary take place on Debye length $\lambda$ [5]. Along with other characteristic lengths (see below for details) conditions (1), (2) are valid when the system of inequalities is fulfilled

$$
\begin{gathered}
R>>h \geq d \geq a>>\lambda>l, \\
\lambda^{2}=\frac{\varepsilon_{\mathrm{aq}} T}{8 \pi e^{2} n_{\mathrm{aq}}^{*}}, \quad n_{\mathrm{aq}}^{*} \simeq \alpha_{\mathrm{aq}} n_{\mathrm{aq}}, \quad a=\frac{2 \pi}{k_{0}} .
\end{gathered}
$$

Here $R$ is the plate radius of the flat capacitor of the cell (Fig. 1) used in the experiments [6,7], $h$ is the distance between two plates of capacitor, $d$ is the thickness of the water layer in the capacitor gap, $a \simeq k_{0}^{-1}$ is the so-called capillary constant, $l$ is the width of the transition region on the vapor-liquid boundary, $\lambda$ is the Debye length, $\alpha_{a q}$ is the water dissociation constant, $n_{\mathrm{aq}}$ is the water density, $T$ is the temperature. Values 


$$
\begin{gathered}
R \simeq 6 \mathrm{~cm}, \quad h \sim(2-3) d, \quad d \sim(1-5) a, a^{2} \simeq 10^{-1} \mathrm{~cm}^{2}, \\
\lambda \sim 10^{-3}-10^{-4} \mathrm{~cm}, \quad l \geq 10^{-8} \mathrm{~cm}
\end{gathered}
$$

indicate that inequalities (3) are fulfilled completely.

The formal consequence of metallization of the free boundary is changing boundary condition in the electrostatics of phenomenon. Instead of the requirement of continuous induction $E_{0}=\varepsilon E_{\text {insid }}$, on the oscillating liquid boundary an electrostatic equipotentiality condition occurs along this surface excited by small-amplitude oscillations. The spectrum of the surface excitations suggests a loss of stability in an external electric field $E_{0}=E_{\text {met }}$ [3] with $E_{\text {met }}$ from (2). In accordance with (1), (2), as external field $E_{0}(t)$ increases, threshold (1) is preceded by critical point (2). As a result, the dielectric surface loses its stability not as a liquid dielectric rather as a good conductor due to "metallization" of the boundary condition in the problem of stability of the vapor-liquid interface. As it is seen, the stability threshold does not depend on the method of electric field shielding, at least till the characteristic scale of attenuation of the electric field deep into flat surface $\lambda$ is significantly less than capillary length $a$. In this case the threshold is determined exclusively by the value of the force acting per unit flat surface area.

The ratio of fields (1), (2) for the water-vacuum boundary

$$
\frac{E_{\mathrm{aq}}^{2}}{E_{\mathrm{met}}^{2}} \simeq\left(1+\frac{3}{\varepsilon_{\mathrm{aq}}}\right)>1, \quad \varepsilon_{\mathrm{aq}}>>1,
$$

where $E_{\mathrm{aq}}^{2}$ is defined by expression (1) with dielectric constant for water, $\varepsilon=\varepsilon_{\mathrm{aq}}$. Note that according to [8], $\varepsilon_{\mathrm{aq}} \simeq 80>>1$. Thus, for water the threshold $E_{0}=E_{\text {met }}$ precedes dielectric instability at $E_{0}=E_{\varepsilon}$, although to a small extent.

Let us turn to the available experimental data on free water surface stability in electric field applied perpendicular to the interface $[6,7]$. We are considering a liquid layer of thickness $d$ placed in a gap of flat capacitor with distance between two plates $h<<R$ ( $R$ is the plate radius, see Fig. 1). High voltage $V$ is applied between the plates. Feasible charge current through the boundary is registered by electrometer 4 . In experiment the control of possible cell

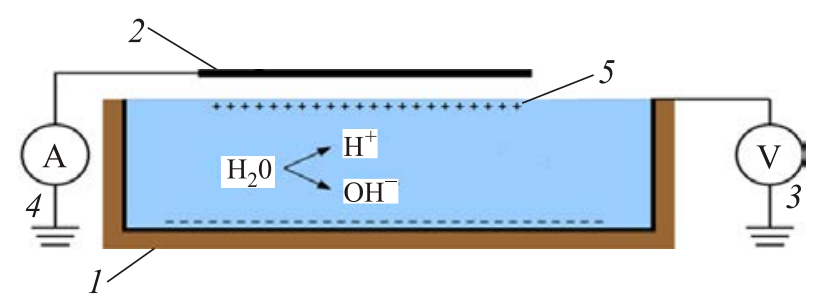

Fig. 1. Scheme of experiment: 1 is the cup (lower capacitor plate), 2 is the collector (upper plate), 3 is the high-voltage source, 4 is the electrometer, 5 is the surface water. tilt and of changing liquid level with respect to the plates under the action of ponderomotive forces of electrostatic origin is provided. Visual observations of the surface state in the horizontal direction enable to monitor breakdown development. Adjusting the voltage $V$ allows us to smoothly approach the value at which the interface instability develops.

The critical conditions were determined by the authors of $[6,7]$ under the assumption of the metallic behavior of the liquid boundary. In the massive case $d>>a$ critical conditions coincide with (2) without references to [8]. Occurrence of current through the interface is identified with loss of boundary the stability. The measurements carried out in [6,7] preceded works [1,2] and were performed for mechanism (2) over a wide range of thickness $d$. The data obtained are in good agreement with predictions (2) including the strong dependence of the critical parameters of the liquid film on its thickness in the range $d<a$. It should be noted that the authors of $[6,7]$ could have paid no attention to the difference between thresholds (1) and (2) because, in accordance with (4), this difference is of the order of several percent. Yet, the details of the discharge of the liquid boundary losing its stability are qualitatively different in dielectric, metallic or weakly conducting states.

When the dielectric boundary loses its stability under the action of electric field $V / h$ in accordance with (1), ceases to be perfectly smooth. The surface oscillation amplitude increasing with time leads to formation of drops of different shape and size of the order of capillary length $a$. Although, by and large, the drops remain neutral, they cannot move far from the geometric position of the free boundary. The charge transfer over the boundary to the upper electrode with the participation of forming neutral drops is equal to zero.

As in the case of dielectric, in critical situation (2) the metallic boundary starts to progressively deform getting into a "boiling" phase with formation of charged drops (streams). These liquid fragments are immediately carried away by the strong electric field to the upper plate (inlet header) of the capacitor. The discharge current occurring threshold-like is further maintained at some stationary level due to the continuous charge supply from the bulk of conductor. The metallic boundary cannot recover itself from the critical state.

The water surface is charged by its own (third) method. The electrometer connected to the upper plate of capacitor registered sharp (time-dependent $\tau_{\text {jet }}$ ) current bursts (events) separated by relatively long intervals $T_{*}>>\tau_{\text {jet }}$ without any charge transfer. Parallel high-speed filming [2] reveals appearance of separate charged streams of diameter $R_{\text {jet }}<<a$ with a very high current density and lifetime of the order of several microseconds so that

$$
\tau_{\text {jet }}^{\text {aq }}=10^{-5}-10^{-4} \mathrm{~s}, \quad \text { at } T_{*}^{\mathrm{aq}} / \tau_{\text {jet }}^{\mathrm{aq}} \sim 10^{3}-10^{4} .
$$


An elementary breakdown act is accompanied by arcing (lightning) and sonic pops (thunder). Judging by the further tranquil state of the boundary (it is literally said that over the 50-ft length of the exposed film only a single frame shows a distinct eruption image), in time $\tau_{\text {jet }}$ the stream carries to the receiver electrode practically the whole charge stored in intercharge gap $T_{*}^{\text {aq }}$ in the accumulation dielectric layer.

Our understanding of behavior of the surface of the liquid dielectric in an external field allows making some specific conclusions. First of all, we can talk about a new, collective electrical mechanism of dielectric breakdown. Along with the famous thermal version of this phenomenon, based on the activation dependence of the conductivity of the dielectric on the temperature [9], it is necessary to take into account a finite probability of formation on the borders of the metal-insulator ion accumulation layers in consequence of limb contact resistance which open the prospects for studying the stability of thin films of normal fluid in the zone of action of forces van der Waals forces. Such experiments for normal liquid films are not yet available.

The most interesting possibility is to detect phenomena characterizing water as the electrolyte. We are talking about its ability to serve as a source of ions to support permanent instability at the liquid-vapor boundary. Expected in conditions (5) intermittent behavior of full charge $Q$ crossing the liquid-vapor boundary has already been observed by one of the authors [10] on the free surface of liquid hydrogen in the presence of artificial (radioactive) source of formation of ions in the volume of liquid. Experiment with water, obviously does not require radioactive pill, but the test, of course, should be implemented.

The cell presented in Fig. 1 is interesting methodically from the point of view of the measurements of low conductivity of the electrolyte because there is no need to control boundaries of the metal-electrolyte.

\section{Experimental technique}

The scheme of experiment is shown in Fig. 1. The relaxation oscillations of charges $Q$ were observed using a cell composed of a metal cup of depth $h=20 \mathrm{~mm}$ and inner diameter $d=47 \mathrm{~mm}$ and an upper plate of diameter $d=15$ мm placed above the cup. The cup was filled with purified water up to the edges. The distance between the water surface and the upper electrode was about $0.5 \mathrm{~mm}$. Surface of water and upper plate formed a flat capacitor. The cell was placed into a glove box filled with gaseous nitrogen. Note, that in our experiments we investigated charge transfer through the water-gaseous nitrogen interface. Constant positive voltage $U$ in the range from 600 to $1700 \mathrm{~V}$ was applied to the metal cup to study the positive charge transfer through the liquid surface. An electrometer was used to measure the value of the charge coming to the upper plate after switching on constant voltage. The recording time was from 10 to $1000 \mathrm{~s}$.

As voltage is applied, the positive charges are pressed by the electric field to the liquid surface while the negative charges leave the bulk through the metal cup. At low voltages, $E_{0} \leq E_{\text {met }}$, slow capacitor charging (renormalization) is observed, and at voltages above $E_{0} \geq E_{\text {met }}$ the charged liquid surface loses stability with registered discharge.

\section{Results}

In Fig. 2 results of measurements of the total charge $Q$ on the upper plate after switching on the voltage as a function of time are shown. These results reflect the development over time the renormalization of capacity of cell occurring as a result of the screening of the external field by charges on the water layer of thickness $h$. The measurements of time dependence were carried out at three constant positive voltage $U<U_{C}$ :

$$
U_{1}=600 \mathrm{~V}, \quad U_{2}=700 \mathrm{~V}, U_{3}=800 \mathrm{~V} .
$$

The critical voltage at which the surface is discharged equals $900 \mathrm{~V}$.

According to the above reasons, the initial capacity of the cell is determined by the distances between the two metal plates: a vacuum gap and the water film thickness. When the voltage is switched on an accumulation layer on the free surface of the water is formed shielding external field $E$ in the liquid. As a result when a experimental curve reaches its plateau the electric field in water is screened completely and capacity of experimental cell or total charge is approaching to its renormalized value. The data show that the asymptotic behavior of the curves is sensitive to the value of voltage. We can propose that the gap does not remain constant, independent on the $U$. Arising on the vapor-liquid accumulation layer is pushing the surface of

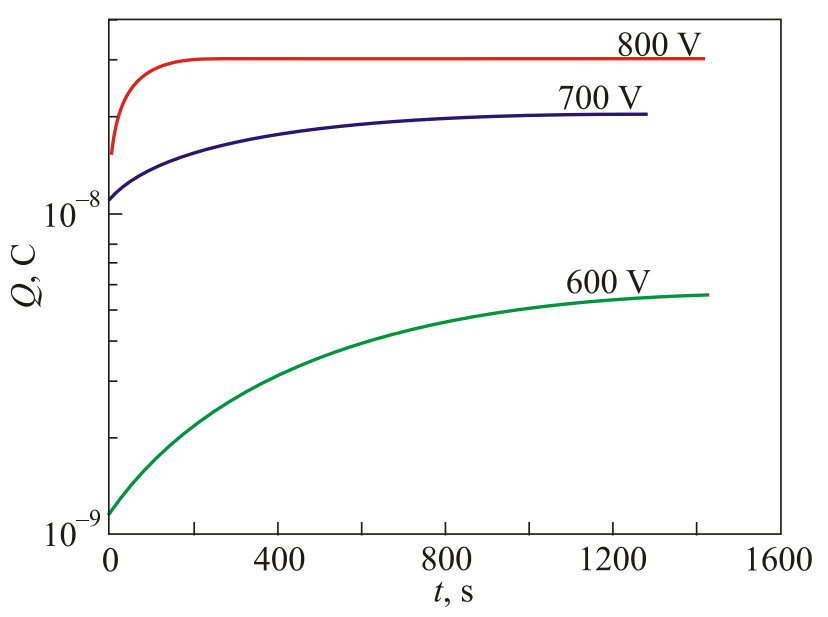

Fig. 2. Time dependence of the charge arriving at the collector after switching on positive dc voltage, $U=600,700,800 \mathrm{~V}$. 


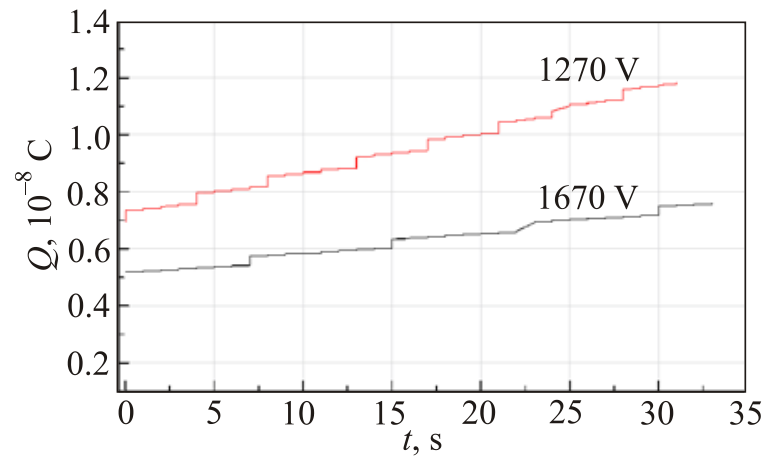

Fig. 3. Time dependence of the charge arriving at the collector after switching on positive dc voltage, $U=1270,1670 \mathrm{~V}$.

water upwards decreasing the gap. This shift of water surface in addition to process of charging is registered in our experiments. As it is seen in Fig. 2 the characteristic time of process of charging is order of $100 \mathrm{~s}$.

As the voltage applied to the cup exceeds critical value $U_{c}$ penetration of charges through the water surface is observed. The $Q$ curve exhibits periodic steps (discontinuities) caused by the charge transfer through the water surface. The time $T_{*}^{\mathrm{aq}}$ between two surface discharges is about 5-10 s and decreases with increase of the applied voltage. The scale of this time is the same as in [6] (see the definitions (5)).

Figure 3 demonstrates two dependences obtained at constant voltages 1270 and $1670 \mathrm{~V}$. It is seen that upon surface discharge approximately $2 \cdot 10^{-10} \mathrm{C}$ arrive at the upper plate, which corresponds to $10^{9}$ particles with a charge equal to the electron charge. Large steps correspond to the process of discharge. Small steps on the curves correspond to discrete sampling data.

\section{Conclusion}

In this paper we discussed the conditions for development of the instability of the liquid surface in an external electric field applied perpendicular to the boundary. It is shown theoretically that the scenario of development of instability is very sensitive to the presence of own charge carriers - ions in the bulk liquid. In the case of pure water is realized the metal scenario. It was shown experimentally that at voltages $U<900 \mathrm{~V}$ applied to the lower plate, the surface of water is charged with a characteristic time of the order of $10^{2} \mathrm{~s}$. At higher voltage $U>900 \mathrm{~V}$ the surface is discharged periodically. The time span between two successive discharges of the surface decreases with increasing applied voltage $U$.

The authors are grateful to Prof. Leonid P. MezhovDeglin for valuable discussions. This work was supported by the Russian Science Foundation, grant No. 14-22-00259.

1. V. Zaitsev and M. Shliomis, PAS USSR 188, 1261 (1969).

2. M. Shliomis, Usp. Fiz. Nauk 112, 427 (1974).

3. Y. Frenkel, JETF 6, 347 (1936).

4. L.D. Landau and E.M. Lifshitz, Electrodynamics of Continuous Media, Vol. 8, Course of Theoretical Physics, Pergamon Press (1960).

5. L.D. Landau and E.M. Lifshitz, Statistical Physics, Third Edition, Part 1, Vol. 5, Course of Theoretical Physics, Pergamon Press (1995).

6. G. Teylor and A. McEvan, J. Fluid Mech. 22, 1 (1965).

7. G. Teylor, Proc. Roy. Soc. A 280, 1383 (1964).

8. R. Robinson and R. Stokes, Electrolyte Solutions, Butterworths Scientific Publications, London (1959).

9. G. Skanavi, Physics of Dielectrics (Strong Fields), Moscow State Technical Press (1958).

10. A.A. Levchenko and L.P. Mezhov-Deglin, Fiz. Nizk. Temp. 22, 46 (1996) [Low Temp. Phys. 22, 33 (1996)]. 